\title{
Brief report \\ Neuroimaging of serotonin transporters in post-stroke pathological crying
}

\author{
Toshiya Murai ${ }^{\mathrm{a}, \mathrm{b}}$, Henryk Barthel ${ }^{\mathrm{c}}$, Jörg Berrouschot ${ }^{\mathrm{d}}$, Dietlind Sorger ${ }^{\mathrm{c}}$, \\ D. Yves von Cramon ${ }^{\mathrm{a}, \mathrm{e}}$, Ulrich Müller, ${ }^{\mathrm{f}, *}$ \\ ${ }^{a}$ Max-Planck-Institute of Cognitive Neuroscience, Stephanstr. 1, D-04103 Leipzig, Germany \\ ${ }^{\mathrm{b}}$ Department of Neuropsychiatry, Kyoto University Hospital, 54 Shogoin-Kawaharacho, Kyoto 606, Japan \\ ${ }^{\mathrm{c}}$ Department of Nuclear Medicine, Leipzig University Hospital, Liebigstr. 20, D-04103 Leipzig, Germany \\ ${ }^{\mathrm{d}}$ Department of Neurology, Leipzig University Hospital, Liebigstr. 22a, D-04103 Leipzig, Germany

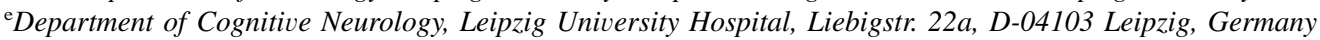 \\ ${ }^{\mathrm{f}}$ Department of Psychiatry, Leipzig University Hospital, Liebigstr. 20, D-04103 Leipzig, Germany
}

Received 6 February 2001; received in revised form 9 July 2002; accepted 28 August 2002

\begin{abstract}
Pathological crying (PC) is a neuropsychiatric disorder characterized by an excessive tendency towards crying after brain damage. To elucidate the role of serotonin neurotransmission for PC, a pilot study was performed using single photon emission computed tomography with [ $\left.{ }^{123} \mathrm{I}\right] \beta$-CIT to estimate central (midbrain/pons and thalamus/ hypothalamus) serotonin transporter (SERT) densities in 15 stroke patients who did or did not have PC. SERT binding ratios in midbrain/pons were significantly lower in the PC subgroup.
\end{abstract}

(c) 2003 Elsevier Ireland Ltd. All rights reserved.

Keywords: Single photon emission computed tomography; $\left[{ }^{123} \mathrm{I}\right] \beta-\mathrm{CIT}$; Serotonin; Brainstem; Stroke

\section{Introduction}

Pathological crying (PC), which is characterized by involuntary and uncontrollable outbursts of crying, is a frequent finding in neurological patients (Allman et al., 1990). Recent studies showed that selective serotonin reuptake inhibitors (SSRIs) are effective in treating PC with minimal side effects (Müller et al., 1999). Since low doses

*Corresponding author. Tel.: +44-1223-333535; fax: +441223-333564.

E-mail address: um207@cam.ac.uk (U. Müller). of SSRIs are very effective, disturbed serotonin neurotransmission has been suggested to be the underlying pathophysiology of PC.

While some stroke patients show PC, others with similar lesion locations do not develop it. Thus, in the present study, we aimed to clarify whether differences in serotonin neurotransmission explain the difference between PC patients with unilateral cerebral lesions and non-PC patients with similar lesions. As a marker of central serotonin neurotransmission, we focused on serotonin transporters (SERTs), since cerebral SERTs have 
recently been shown to be reduced in several psychiatric disorders with disturbances of impulsiveness and emotion (Tiihonen et al., 1997; Heinz et al., 1998; Malison et al., 1998; Willeit et al., 2000). To estimate regional cerebral SERT density, we used a single-photon emission computed tomography (SPECT) radioligand, $\left[{ }^{123} \mathrm{I}\right] 2 \beta$-carbomethoxy-3 $\beta$-(4-iodophenyl)tropane $\quad\left(\left[{ }^{123} \mathrm{I}\right] \beta\right.$ CIT), which has high affinity to SERTs (Neumeyer et al., 1991).

\section{Methods}

\subsection{Subjects and clinical ratings}

The present study was approved by the ethics committee of the University of Leipzig. All patients gave written informed consent to participate in this study after the procedure had been fully explained. Six stroke patients with PC and nine stroke patients without PC were recruited from the Stroke Unit of the Department of Neurology, University of Leipzig. All patients had single and unilateral cerebral ischemic infarctions. Patients with moderate to severe language comprehension or cognitive deficits, with a history of psychiatric diseases, or with current psychotropic drug use were excluded. The existence of PC was evaluated using a questionnaire developed by Moore et al. (1997). Patients were regarded as suffering from PC when the following three conditions were fulfilled: (a) crying episodes were provoked more easily than in the pre-stroke period; (b) crying episodes were (at least sometimes) provoked suddenly for little or no reason; (c) crying episodes were (at least sometimes) uncontrollable. The global severity of PC was determined using a 6-point scale (Allman et al., 1992).

\subsection{Neuroimaging and data analysis}

$\left[{ }^{123} \mathrm{I}\right] \beta-\mathrm{CIT}$ SPECT was performed within one week of the clinical assessment in all patients. A SPECT scan was acquired $24 \mathrm{~h}$ after injection of $185-200 \mathrm{MBq}\left[{ }^{123} \mathrm{I}\right] \beta-\mathrm{CIT}$ using a brain-dedicated camera (CERASPECT 3000, Digital Scintigraphics Inc., Waltham, MA) with three rotating collimators (Holman et al., 1990). Within $30 \mathrm{~min}$ $\left(360^{\circ}\right.$ rotation, 120 projections $), 1-2$ million counts were collected in a $128 \times 128 \times 64$ matrix. Images were reconstructed using standardized filtered back-projection with a 2D-Hanning Filter (cutoff 0.5). Images were corrected for attenuation by Chang's first order method (attenuation coefficient $\mu=0.15 \mathrm{~cm}^{-1}$ ). The spatial resolution in the final images was approximately $8 \mathrm{~mm}$ in all spatial dimensions as previously described (Bettin et al., 1997).

Regions of interest (ROI) analysis was performed on a UNIX workstation using a commercial software package (HERMES/MultiModality, Nuclear Diagnostics, Hägersten, Sweden) by an experienced examiner without knowledge of the diagnoses of the subjects. The voxels of interest (VOIs) were defined on a representative 3-D magnetic resonance imaging (MRI) scan obtained from a healthy subject. The VOIs included a column-shaped VOI for midbrain/pons $\left(3.3 \mathrm{~cm}^{3}\right)$, a column-shaped VOI for thalamus/hypothalamus $\left(6.9 \mathrm{~cm}^{3}\right)$, and irregular VOIs for left or right cerebellar hemispheres $\left(2.0 \mathrm{~cm}^{3}\right)$. Each SPECT image was manually coregistered to this reference MRI by shifting, rotating and resizing SPECT images three-dimensionally, using internal (striatal activity and brainstem activity) and external (e.g. cerebral longitudinal fistula, lateral sulcus and cerebellum) landmarks. Then, the pre-defined VOIs were transferred to the coregistered SPECT images, and the average count densities (counts/ pixel) were calculated for each VOI. In the present study, cerebellar activity ipsilateral to the stroke side was used as a reference region, to avoid the possible effect of decreased non-specific binding due to decreased perfusion caused by crossed cerebellar diaschisis. Ratios between the count density in target regions (midbrain/pons, thalamus/hypothalamus) and cerebellum (ipsilateral to the stroke side) were calculated. This ratio minus 1 represents the specific/non-displaceable binding ratio and was assumed to be directly related to SERT availability at the time of the binding equilibrium.

In addition, $\left[{ }^{123} \mathrm{I}\right] \beta-\mathrm{CIT}$ SPECT findings from 10 subjects who were age-matched to both patient groups were selected from our control data pool (mean age: $61.5 \pm 10.9$ years, 6 women). The 
Table 1

Demographic, lesion and clinical characteristics of the study population

\begin{tabular}{lll}
\hline & PC group & Non-PC group \\
\hline No. of patients & 6 & 9 \\
Age, mean (S.D.) & $60.2(11.0)$ & $60.4(10.9)$ \\
Sex (M:F) & $2: 4$ & $4: 5$ \\
Days after stroke onset, mean (S.D.) & $6.22(2.39)$ & $8.67(5.89)$ \\
(SPECT examination) & & $8: 1$ \\
Lesion side (R:L) & $4: 2$ & \\
Lesion location & & 6 \\
$\quad$ Lateral frontal & 4 & 0 \\
$\quad$ Medial frontal & 0 & 5 \\
$\quad$ Parietal & 3 & 4 \\
Temporal & 2 & 0 \\
Occipital & 0 & 4 \\
Striatum & 3 & 0 \\
Thalamus & 0 & 9 \\
Hemiparesis & 5 & 6 \\
Sensory deficit & 5 & 1 \\
Mild aphasia & 4 & 4 \\
Visuospatial deficit & 2 & $25.7(2.4)$ \\
MMSE (max =30), mean (S.D.) & 2 & $6.2(6.9)$ \\
HDRS (max =67), mean (S.D.) & $24.2(3.1)$ & 0 \\
Pathological laughing & $4.3(2.9)$ & \\
\hline
\end{tabular}

subjects had been screened by neurologists and shown to be without evidence of neurological or major psychiatric diseases. The SPECT data were analyzed with the same ROI method described above.

Statistical differences in SPECT measurements were assessed by two-tailed Mann-Whitney $U$ tests. Spearman rank correlations were used for the correlations between SPECT measurements and global severity of PC.

\section{Results}

Table 1 summarizes the demographic, pathoanatomical and clinical features of the study population. The PC group and the non-PC group did not differ significantly in age, gender, duration between stroke event and SPECT examination, laterality of the lesion, or lesion location within the damaged hemisphere. There was no difference in the involvement of hemiparesis, sensory deficits, language disorders, or visuospatial deficits. No significant difference was found in scores on the
Hamilton Depression Rating Scale (HDRS) or Mini-Mental Status Examination between the two groups. Only one patient (in the non-PC group) was diagnosed as suffering from post-stroke depression $(\mathrm{HDRS}=23)$.

Midbrain/pons $\left[{ }^{123} \mathrm{I}\right] \beta$-CIT binding ratios of the PC group were significantly lower than those of the non-PC group $(1.26 \pm 0.51$ vs. $1.93 \pm 0.59, P=$ 0.025 , Fig. 1). One patient with PC showed an extremely low midbrain/pons binding ratio. After removal of this outlier, a strong trend remained $(P=0.053)$.

No significant difference was found in the thalamus/hypothalamus binding ratios $(2.91 \pm 1.29 \mathrm{vs}$. $3.61 \pm 0.90, P=0.29)$. In both investigated regions, the binding ratios of the non-stroke control group were not significantly different from those of the PC group $(1.79 \pm 0.63$ vs. $1.26 \pm 0.51$ for midbrain/pons, $3.55 \pm 0.48$ vs. $2.91 \pm 1.29$ for thalamus/hypothalamus).

For the whole stroke group $(n=15)$, the correlation of the global severity of PC with midbrain/ pons binding ratios was significant $(r=-0.60$, 


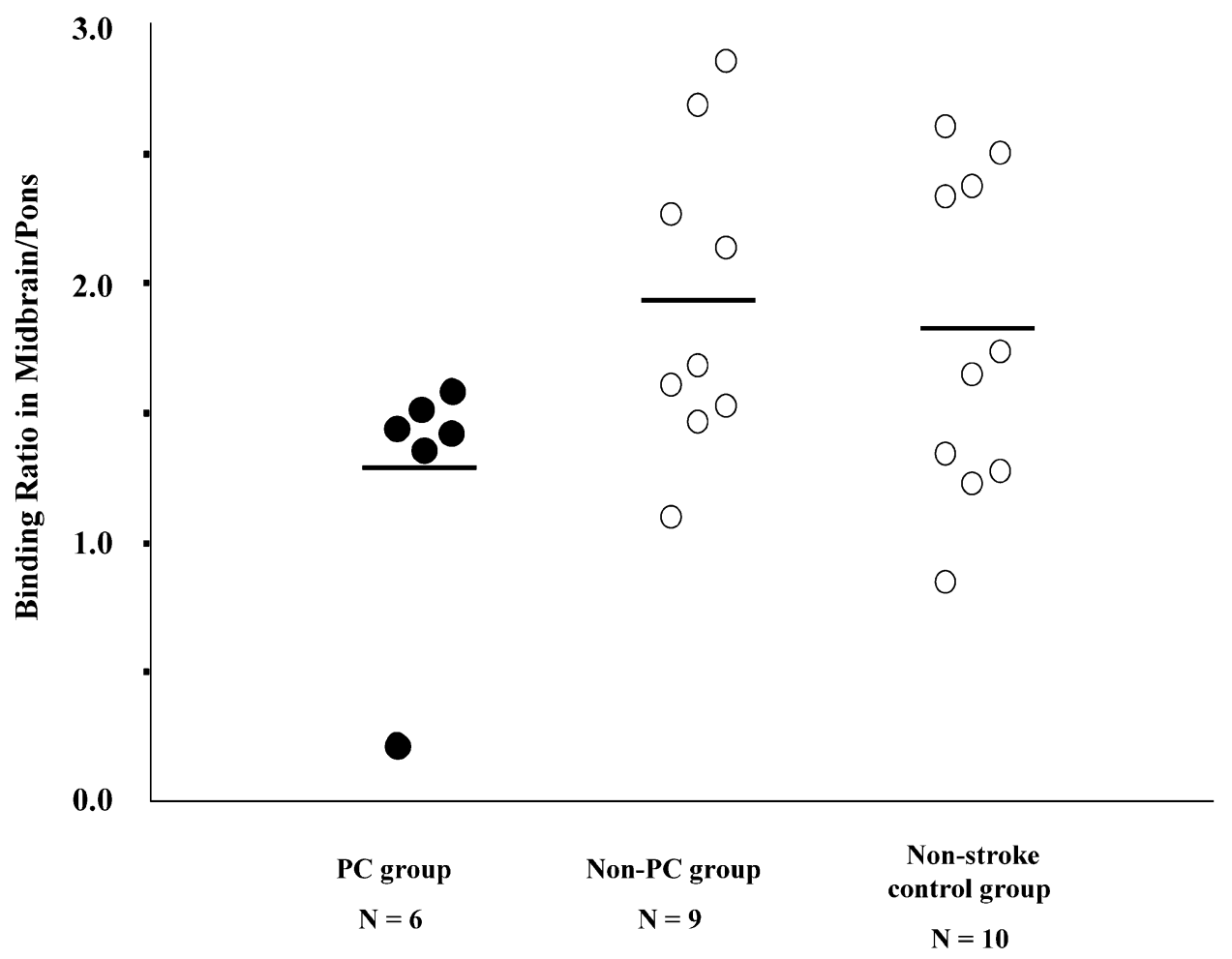

Fig. 1. Midbrain/pons $\left.{ }^{123} \mathrm{I}\right] \beta-\mathrm{CIT}$ binding ratio in stroke patients with $\mathrm{PC}$, stroke patients without PC, and non-stroke control subjects.

$P=0.019$ ), while that with thalamus/hypothalamus binding ratios was not significant $(r=-0.39$, $P=0.16)$.

\section{Discussion}

We examined midbrain/pons and thalamus/ hypothalamus SERT density in stroke patients both with and without PC, and found that midbrain/ pons SERT density was lower in patients with PC than in patients without PC. The major limitation of this study is its relatively small sample size; the findings therefore have to be considered as preliminary.

Deficits in serotonin neurotransmission have been linked to impulsive and disinhibitory behavior in animal studies (Robbins, 1997) and human studies (Evans et al., 2000). From the current results, considering the uncontrollable nature of crying in PC, it was suggested that post-stroke PC is a variant of impulsive/disinhibitory behavioral symptoms modulated by the mesocortical serotonin system.

It is not clear why SERT density is reduced in post-stroke PC patients. Brainstem SERTs may be down-regulated in PC patients as a secondary reaction to the stroke, for example, as an adaptive reaction to minimize the global perturbation of neurotransmission caused by the cerebral lesion. However, considering that there was no major difference in lesion location between the PC and non-PC groups, this hypothesis would not explain why brainstem SERTs are reduced only in the PC group. Another explanation might be that the amount of SERT expression was already at low levels in the PC group before the stroke events. No patient in the present study had a history of depression or apparent emotional lability before the onset of stroke. Thus, the low SERT density in the brainstem, discovered in the PC group in 
the present study, would be considered a latent vulnerability, which determines the higher susceptibility to PC after stroke. In good accordance with this interpretation is the distribution of the midbrain/pons SERT density measurements of nonstroke controls, which largely overlapped those of the combined group of PC and non-PC patients (Fig. 1). In other words, a normal population is variable in raphe SERT density and lower individual levels of raphe SERT expression might affect the susceptibility to PC if a stroke happened to occur.

In summary, this neuroimaging study suggests altered serotonin neurotransmission in patients with post-stroke PC. Further studies are necessary to elucidate the pathophysiology of PC and other disorders of emotional control.

\section{Acknowledgments}

This work was supported by DAAD Bonn and by BMB7 IZKF Leipzig (Projects C-8 and C-9).

\section{References}

Allman, P., Hope, R.A., Fairburn, C.G., 1990. Emotionalism following brain damage: a complex phenomenon. Postgraduate Medical Journal 66, 818-821.

Allman, P., Marshall, M., Hope, T., Fairburn, C., 1992. Emotionalism following stroke: development and reliability of a semi-structured interview. International Journal of Methods in Psychiatric Research 2, 125-131.

Bettin, S., Kämpfer, I., Seese, A., Schäfer, A., Reuter, M., Lössner, J., Dietrich, J., Wagner, A., Knapp, W.H., 1997. Striatal uptake of I-123- $\beta$-CIT and I-123-IBZM in patients with extrapyramidal symptoms. Nuklearmedizin 36, 167-172.

Evans, J., Platts, H., Lightman, S., Nutt, D., 2000. Impulsiveness and the prolactin response to $d$-fenfluramine. Psychopharmacology 149, 147-152.
Heinz, A., Ragan, P., Jones, D.W., Hommer, D., Williams, W., Knable, M.B., Gorey, J.G., Doty, L., Geyer, C., Lee, K.S., Coppola, R., Weinberger, D.R., Linnoila, M., 1998. Reduced central serotonin transporters in alcoholism. American Journal of Psychiatry 155, 1544-1549.

Holman, B.L., Carvalho, P.A., Zimmerman, R.E., Johnson, K.A., Tumeh, S.S., Smith, A.P., Genna, S., 1990. Brain perfusion SPECT using an annular single crystal camera: initial clinical experience. Journal of Nuclear Medicine 31, 1456-1461.

Malison, R.T., Price, L.H., Berman, R., van Dyck, C.H., Pelton, G.H., Carpenter, L., Sanacora, G., Owens, M.J., Nemeroff, C.B., Rajeevan, N., Baldwin, R.M., Seibyl, J.P., Innis, R.B., Charney, D.S., 1998. Reduced brain serotonin transporter availability in major depression as measured by $\left[{ }^{123} \mathrm{I}\right]-2 \beta-$ carbomethoxy-3 $\beta$-(4-iodophenyl)tropane and single photon emission computed tomography. Biological Psychiatry 44, 1090-1098.

Moore, S.R., Gresham, L.S., Bromberg, M.B., Kasarkis, E.J., Smith, R.A., 1997. A self report measure of affective lability. Journal of Neurology, Neurosurgery and Psychiatry 63, 89-93.

Müller, U., Murai, T., Bauer-Wittmund, T., von Cramon, D.Y., 1999. Paroxetine versus citalopram treatment of pathological crying after brain injury. Brain Injury 13, 805-811.

Neumeyer, J.L., Wang, S., Milius, R.A., Baldwin, R.M., ZeaPonce, Y., Hoffer, P.B., Sybirska, E., Al-Tikriti, M., Charney, D.S., Malison, R.T., Laruelle, M., Innis, R.B., 1991. $\left[{ }^{123} \mathrm{I}\right]-2 \beta$-Carbomethoxy-3 $\beta$-(4-iodophenyl)-tropane $(\beta$ CIT): high affinity SPECT radiotracer of monoamine reuptake sites in brain. Journal of Medicinal Chemistry 34, 3144-3146.

Robbins, T.W., 1997. Arousal systems and attentional processes. Biological Psychology 45, 57-71.

Tiihonen, J., Kuikka, J.T., Bergström, K.A., Karhu, J., Viinamäki, H., Lehtonen, J., Hallikainen, T., Yang, J., Hakola, P., 1997. Single-photon emission tomography imaging of monoamine transporters in impulsive violent behaviour. European Journal of Nuclear Medicine 24, 1253-1260.

Willeit, M., Praschak-Rieder, N., Neumeister, A., Pirker, W., Asenbaum, S., Vitouch, O., Tauscher, J., Hilger, E., Stastny, J., Brücke, T., Kasper, S., 2000. [ $\left.{ }^{123} \mathrm{I}\right]-\beta$-CIT SPECT imaging shows reduced brain serotonin transporter availability in drug-free depressed patients with seasonal affective disorder. Biological Psychiatry 47, 482-489. 